\title{
Endocrine Problems in Children and Adolescents Who Have Disabilities
}

\author{
Margaret Zacharin \\ Department of Endocrinology, and Murdoch Children's Research Institute, Royal Children's Hospital, \\ Parkville, Vic., Australia
}

\section{Key Words}

Body size assessment · Bone mass accrual · Bone quality assessment - Management of puberty - Pubertal problems . Pubertal status $\cdot$ Salt and water balance

\begin{abstract}
The consequences of disability span almost all aspects of life, for families and for an affected individual. Endocrine aspects of disability cover disorders of hypothalamic pituitary dysfunction, bone health, puberty, fertility, sexual function and contraceptive needs. Adequate endocrine assessment for a child with a disability requires extensive and sensitive history to address social issues, general nutrition, vitamin D status, specific hypothalamic pituitary problems, together with parental concerns regarding puberty and contraception. Bone health assessment includes history of minimal trauma fracture, need for intervention or fitness for surgery. Strategic interventions to improve outcome include management of puberty, vitamin D status, biomechanical stimulation with vibration and standing and possible consideration for bisphosphonate in special circumstances. Both early and late puberty cause major family concerns. The paediatrician must be aware of a range of management options to improve outcomes for the child and family.
\end{abstract}

(c) 2013 S. Karger AG, Basel

\section{Background}

Current estimates from the World Health Organization estimate at least 100 million people to currently suffer moderate to severe disability [1]. A United States Bureau of Census report from 2004 found in excess of 32 million disabled adults and 5 million children with disabilities aged less than 18 years. The consequences of disability span almost all aspects of life, for both families and the affected individual.

Endocrine aspects of disability cover disorders of hypothalamic pituitary dysfunction, bone health and in girls, difficult problems of menstrual management. Sexual function and fertility issues may relate specifically to the type of disability, requiring individualization of management strategies [2]. Hypothalamic pituitary disorders include impairment of growth, ACTH and TSH production, diabetes insipidus and disorders of thirst perception and regulation. Pubertal disorders span central precocious puberty, particularly in the presence of midline defects, premature adrenarche with neural tube defects, and delayed or arrested puberty.

Endocrine assessment may reveal a surprising amount of pathology. Reasons for referral often relate to sudden, apparent changes in pubertal status and consequent increase in parental anxiety, an added burden to an already

\section{KARGER}

E-Mail karger@karger.com

www.karger.com/hrp
(C) 2013 S. Karger AG, Basel

1663-2818/13/0804-0221\$38.00/0
Margaret Zacharin

Department of Endocrinology

Royal Children's Hospital

Flemington Rd., Parkville, VIC 3052 (Australia)

E-Mail margaret.zacharin@ rch.org.au 
distressed family. A first endocrine consultation can thus be fraught with difficulty, sometimes with a distressed and perhaps angry parent and a non-verbal child.

For the present paper the Medline database was searched for all articles related to disability.

\section{Clinical Assessment}

Provision of adequate endocrine assessment and care for a child with a disability requires extensive and sensitive history-taking. Fears and perceptions of the parents must be explored if a satisfactory outcome is to be achieved. Issues of birth trauma, familial, consanguineous or genetic causes for disability may be associated with unexpressed guilt. Particular stressors such as single-parent status, lack of family support and financial difficulties all impinge upon ability to cope. Identification of major carers should be established.

Regardless of the specific circumstance of the child, a family history which includes pubertal timing, heights of siblings and parents is essential to set the scene for expectations within a family.

\section{Specific Questions That May Differ from a Standard Consultation}

Linear growth is usually not a major concern for families when disability is profound. However, rapid growth of recent onset is associated with onset of puberty and may cause significant concern, both in terms of family expectation for final height outcome and with consequent need for changes in medical equipment, such as provision of an adequately sized wheelchair, scoliosis bracing or ankle-foot orthoses. It is uncommon for parents to wish for to maximize final height, even if growth hormone deficiency is detected, due to increasing difficulties with size, mobility and transfer.

Early adrenarche with pubarche is common in children with neural tube defects, hydrocephalus and spina bifida, often occurring as early as age 3-4 years but not necessarily associated with ongoing progress into puberty. It causes major parental anxiety, particularly with hygiene management in a child with continence issues. Practical management strategies can be extremely useful to reduce parental anxiety.

Hypoglycaemia secondary to ACTH or growth hormone deficiency may go unrecognized in the presence of a complex disorder with a midline defect. These problems are more likely to be missed than in a healthy child. Repeated hypoglycaemia adversely impinges upon brain function. Epilepsy is so common with this group of disorders that a precipitating cause such as hypoglycaemia or hypocalcaemia may be missed and if recurrent will contribute to further neuronal loss.

Vitamin D status needs careful assessment, both from a bone health point of view and also in terms of hypocalcaemia risk and thus contribution to epilepsy.

\section{Salt and Water Balance}

Midline defects such as holoprosencephaly can be associated not only with diabetes insipidus but also with disorders of thirst perception, with an altered hypothalamic set point for thirst around a serum sodium of 148$152 \mathrm{mmol} / \mathrm{l}$. In a dehydrated child, with an intercurrent illness, such as respiratory infection or gastroenteritis, a serum sodium of $160-170 \mathrm{mmol} / \mathrm{l}$ may instigate emergency management. If a high hypothalamic set point is not recognised it is extremely easy to overhydrate the child, where aserum sodium of $135-140 \mathrm{mmol} / \mathrm{l}$ will represent water logging and can precipitate cerebral oedema.

Conversely, diabetes insipidus may be mild, to the point of not requiring daily treatment, but decompensation can occur in these children at a time of intercurrent illness and may only require intermittent vasopressin.

Assessment of feeding is essential to assess weight gain, vitamin $\mathrm{D}$ status, use of anticonvulsants and bone health. Almost without exception, percutaneous gastrostomy feeds contain sufficient vitamin $\mathrm{D}$, even in the presence of anticonvulsants. Conversely, severely disabled children who are orally fed are almost universally vitamin $\mathrm{D}$ deficient. A careful history may also reveal minor episodes of aspiration associated with reflux, often missed in consultation.

A multitude of other possible inputs in the disabled child have possible effects on growth, puberty and bone health, including cardiovascular abnormalities, longterm respiratory or inflammatory problems and increasing scoliosis. All these affect the direction and necessity for possible endocrine intervention, such as corticosteroid use resulting in further reduction of an already compromised skeleton. Necessary scoliosis surgery increases pneumonia and respiratory failure risk, but needs to be balanced against current bone quality and likelihood of successful instrumentation. 


\section{Examination of a Child with a Disability}

\section{Body Size Assessment}

- Assessment of appropriate weight for height helps define nutritional deficit versus smallness due to pubertal delay.

- Recognition of height differential in the presence of a spinal cord lesion is pertinent, such as spina bifida or transverse myelitis, where the skeleton below the level of the lesion has limited growth potential, due to immobility.

- Specific musculoskeletal problems such as scoliosis and rickets may be found.

\section{Pubertal Status}

This should be assessed at any age.

- Early adrenarche and pubarche secondary to increasing DHEAS are common with neural tube defects, but not necessarily predictive of central precocious puberty.

- True puberty is common in a child with a major brain malformation [3] and generates huge fears and concerns for the family, in addition to emotional alterations in the child. However, the onset of early puberty in such a child, who also has poor mobility, has benefits, with a positive effect on both bone mineral accrual and on emotional maturity and family interaction.

- Delayed puberty is more common in a very thin child, so nutritional status requires significant attention. Its adverse effect on bone health is doubly significant, by keeping the skeleton in a prepubertal state for unnecessary time. Nevertheless, a family history of pubertal delay is essential, for understanding of the individual. In summary, this complex area includes the necessity to consider general nutrition, vitamin D status, specific hypothalamic pituitary problems, parental concerns regarding puberty, fracture history and risks. Specific attention should be given to diabetes insipidus and an inappropriate set point for hypothalamic thirst detection, risks of low ACTH and the need for the use of intermittent vasopressin. Low thyroxine levels contribute to poor mentation. Growth hormone deficiency probably only requires discussion with the family.

\section{Bone Mass Accrual}

For both children and adults, genetic predisposition determines about $80 \%$ of bone mass, with the remaining $20 \%$ related to nutritional status, calcium and vitamin D intake, general health, body weight, exercise, medication and with input from gonadal hormone status [4].

In all children, cumulative gain in lumbar spine bone mineral density closely parallels the growth curve, with the majority accrual of mineral content and cortical thickness occurring at puberty. At this time in a girl, oestrogen inhibits periosteal apposition whilst simultaneously stimulating endocortical formation, leading to narrowing of the medullary cavity [5]. By contrast, testosterone increases bone size by increasing external bone apposition and simultaneously removing bone from the endocortex, resulting in a wider, more stable structure. In children with a disability, multiple stressors contribute to poor bone health and many points at which an intervention may be considered.

\section{Adverse Effects of Disabilities on Bone Health}

As disability increases, the number of adverse factors for bone health also increases [6]. Growth measurements become extremely inaccurate [7]. Increasing disease severity and duration reduce growth and total bone mass accrual. Later childhood onset of disability causes maximal failure of both cortical bone accrual and trabecular mineralization [8].

A multitude of factors contribute to reduced bone mass. These include poor nutrition, anticonvulsants, reduced mobility $[9,10]$ and endocrine disturbances including vitamin $\mathrm{D}$ deficiency and increased cytokine activity. These in turn impair growth, have direct effects on bone and aggravate reduction in bone health. Vitamin D sufficiency is impaired by many factors, particularly in a child with a disability, including low sun exposure, little time spent outdoors, generally more clothing with reduced skin exposure, both direct and indirect effects of anticonvulsants on bone, all compounding and escalating adverse effects on bone health [11-14]. Those with multiple adverse factors have been shown to be at highest risk [15].

\section{Assessment of Bone Quality}

This can be extremely difficult in a child with a disability.

Bone density (BMD) measurement provides a surrogate measure of bone health, even in a healthy child. In the situation of reduced linear growth, poor height, delayed puberty, anticonvulsant use and possible vitamin D 
deficiency, complicated by kyphoscoliosis, with or without orthopaedic instrumentation, a proper assessment becomes near impossible. BMD measurement of the lumbar spine may be possible, provided the child is carefully positioned but its use is limited by those factors.

If used at all, a volumetric adjustment must be undertaken to account for effects of differences in height from the general population with whom it is being compared [16].

Its main use is as a recurrent measure over a number of years, to ensure that bone accrual is taking place at an appropriate rate with time and, if necessary, with intervention. It is expected that $3-5 \%$ increase per annum of areal $\mathrm{BMD}$ at the lumbar spine should occur in the prepubertal child, with $10-15 \%$ per annum in the pubertal adolescent. For measurement of bone density in the appendicular skeleton it is frequently necessary to use sites other than the femoral neck because the child cannot be recurrently accurately positioned for comparison. The lower femur or radius may be used as alternative sites [17].

Other methodologies for proper assessment of bone health may be required. These include lateral thoracolumbar spine X-ray for crush fractures. Particularly in the presence of spinal rods, an X-ray is of no value. Sometimes a bone scan may be helpful to locate the site of a recent spinal fracture in a child who is unable to communicate verbally but where pain appears to be a possible cause for distress.

Peripheral quantitative computed tomography (pQCT) is an increasingly utilized and valuable assessment for bone health in a child with a disability, as it provides a measurement of cortical thickness and quality. A low pQCT measurement has been demonstrated to be closely associated with and predictive of fracture risk [18]. In a child with cerebral palsy the total cross-sectional area of bone is markedly decreased, along with reduction of cortical cross-sectional area although cortical BMD remains largely unchanged. The smaller, thinner bone, particularly in the prepubertal child, thus remains at major risk of fracture. With immobility, low muscle bulk and reduced muscle 'pull' on bone (the muscle bone unit) [19] cortical accrual is further reduced and the bone inevitably exposed to any inadvertent trauma.

\section{Management of Bone Health}

Biomechanical stimulation of bone using vibration therapy, either with a vibration platform or if available, whole-body vibration platform, have been recently dem- onstrated to be extremely effective, resulting in 3-5\% increase in bone density measurement over 6-12 months $[20,21]$. This should be combined with as much time as possible spent in the weight-bearing position. Many children with major biomechanical disabilities are able, with encouragement and support, to stand for 20-30 min per day in a support frame, also shown to improve bone accrual [22-24].

Bisphosphonate use for management of bone health in cerebral palsy is often requested by orthopaedic surgeons and general paediatricians. Whilst bisphosphonates harden bone, other contributors of calcium and vitamin D status, puberty and use of mechanical and weight-bearing stimulation must be addressed first. The major requirement in these children is the ability to withstand a load without fracture. BMD of the lumbar spine has not been shown to be predictive of future fracture risk [25]. Recent PQCT data is more hopeful in this regard [26]. A balanced decision is therefore needed before their use.

Puberty, by contrast, increases bone mass for life by nearly $50 \%$ in both sexes. As disordered puberty, particularly late puberty, is extremely commonly seen in children with a disability, attention must be given to possible pubertal induction. This action will definitively result in a larger, thicker, stronger bone, more able to withstand any mechanical insult [6].

However, it is an absolute requirement during a consultation for bone health for children and adolescents with a disability that a detailed discussion be undertaken with parents and carers as to some bone health risks that simply cannot be changed.

Whilst biomechanical, pubertal or bisphosphonate interventions will improve bone density and quality and reduce long-term risks, a disabled child has an irremediable increased lifetime risk for fracture [25], based on reduced muscle bulk, poor cushion effect during any fall, a risk of sudden fall or injury on an exposed bone often occurring from a clumsy angle, without an ability of the child to save him- or herself [6].

In addition, any assessment must involve detailed enquiry into the situation where a fall occurred. It is extremely common for bony injury to occur outside of the home, often at school where no history is forthcoming as to the nature of the injury. In a child who has not previously sustained any spontaneous or minimal trauma fracture, this raises an index of suspicion that the fracture may have been traumatic and therefore no major change in medical management strategy is required. Conversely, a long bone fracture requiring increased limitation of mobility in plaster casting or after surgery will inevitably re- 
sult in escalating bone loss in areas around the injured limb and may result in a cascade of increasing local, minimal trauma fractures.

In summary, the endocrinologist is frequently asked to address questions of fractures after plaster casting, assessment of minimal trauma fracture and the need for intervention or fitness for surgery, particularly for spinal instrumentation. These should be addressed with attention to puberty, vitamin D status, biomechanical stimulation with vibration and standing, and with possible consideration for bisphosphonate in special circumstances.

\section{Pubertal Problems}

\section{Precocious Puberty}

This event in a child with severe global developmental disability causes massive family upheaval. The child who is non-verbal may become restless and irritable, with moaning and crying. He or she may appear to be moody and sad, even if communication is severely limited. There may be a history of onset of thelarche or pubarche at a very early age which did not progress for 2-3 years with recent escalation. Almost without exception, the major parental concern for a girl is imminent onset of menstruation.

\section{Parental Concerns}

A first approach to this problem should bear in mind the large number of possibilities for a change in the child's behaviour. Whilst the parent is likely to focus on newly perceived changes of puberty and to direct all of their concerns to this area, there may be a number of other possibilities, including intercurrent events such as urinary tract infection or constipation. Furthermore, a child with a global disability and immobility may have sustained a fracture at any time, either of a long bone or thoracolumbar spine, with severe pain and apparent disturbed mood, possibly inappropriately ascribed to puberty.

Assuming that non-pubertal issues have been sorted out and excluded as a cause for recent change in behaviour and mood, it is necessary to deal with parental anxiety, which comprises a huge component of fear and surprise. Interestingly, many parents of children with disabilities have a profound and erroneous belief that the onset of puberty in a girl presages immediate onset of menstruation. If this very specific misunderstanding can be addressed acutely, it can make a major difference to any subsequent discussion of need for intervention.
We undertook a study of the impact of menstruation in adolescents with disabilities related to cerebral palsy, to gauge significant parental concerns. Some $50 \%$ of families had sought medical advice regarding menstrual management, half of whom had done so before menarche. The majority (75\%) of girls with a disability had a regular monthly cycle, with periods lasting 3-7 days and with little or no pain reported in over half. Three quarters of parents who responded to our questionnaire reported that they were happy with their daughter's puberty [27]. Whilst this is reassuring in terms of normality, the needs of a family remain for adequate discussion and education with regard to their child. A few other studies report similar data [28-30].

\section{Management of Puberty}

An overriding concern of parents is grief for perceived loss of childhood. This is particularly poignant for the family of a child with a major global disability. There are also significant concerns with regard to the child communicating his or her needs and the possibility of pain or discomfort. Family fears include possible deterioration in behavior including masturbation, perceived hygiene difficulties at time of menstruation, difficulties with mobility with increasing body size and with an undercurrent of unexpressed fear related to possible exposure to unwanted sexual contact or to pregnancy [31, 32].

A discussion of puberty needs to include its positive aspects. Explanation of the critical input of puberty to bone health is particularly important where children have multiple risks to bone health. Parents need to understand the contribution of sex hormones to brain maturation with improvement frequently seen in non-verbal communication, eye contact, slightly better comprehension, communication and in some cases, an improved physical ability to assist with locomotion and transfer [31].

Where communication is limited, specific issues need to be considered for the disabled child, even when puberty is at a normal time.

Breast development is normally accompanied by breast tenderness in the early phases of growth. In the child who wears a harness while in a wheelchair, the harness may impinge upon growing breast tissue and can be extremely uncomfortable. Unless specifically pointed out, this is rarely recognized by families.

Hygiene management for a child in nappies, in the presence of increasing amounts of pubic hair, poses significant concerns for families and specifically needs to be addressed, with verbal permission to trim or shave pubic hair of the affected child. This appears to be a significant 
stumbling block for some families unless specifically discussed.

In general, a family needs to be reassured that the average age of menarche is around 12.5 years and therefore immediate menarchal onset is not an issue. However, in a child with a disability it is not uncommon for pubertal progress to be quite intermittent with switch on and off of gonadotrophin and consequent oestrogen. Should this occur, as in the normal adolescent, a small withdrawal bleed may occur in the early stages of puberty. This needs to be specifically discussed with families to allay concern.

Depending on the type of disability, there may be problems of interpreting emotional changes, with apparent frustration, anger or emotional lability. A less severely disabled child, particularly with autism, may demonstrate an extreme pathological fear of blood or occasionally demonstrate sexualized behaviour at an early age, with increasing concerns for families as to risk of sexual contact.

\section{Menstrual Management}

The major concerns of a family with a girl undergoing puberty in the context of disability [33] are the three questions: can we stop periods, will there be side effects, and how will we address contraception? Options for management include the oral contraceptive pill, use of transdermal oestrogen, implantable progestogen or a progestogen-bearing intrauterine device [34, 35].

The oral contraceptive pill is usually first-line treatment, used on a continuous basis by cutting off the lactose-containing pills. Whilst this can result in endometrial atrophy and long-term amenorrhoea, it is not infrequently associated with breakthough, intermenstrual bleeding, particularly in the situation where a child is in care outside of the family or occasionally in the context of intercurrent antibiotic use. Mood change or weight gain can occur with the oral contraceptive pill, as for any user. Anticonvulsants increase hepatic oestrogen excretion rate. In this situation a high-dose $(50 \mu \mathrm{g})$ ethinyl oestradiol-containing pill is needed to be effective. In a child who is severely immobilized in a wheelchair with knees bent, there is at least a theoretical risk of venous thrombosis.

Implantable progestogen: depot progestogen is recognized by most families as a method to successfully induce amenorrhoea. Intramuscular injection every 12 weeks is usually sufficient. However, its mode of action is not only by inducing endometrial atrophy but also by switching off the hypothalamic pituitary ovarian axis with resultant hypoestrogenism $[36,37]$. In both a normal girl and specifically a girl with a disability and immobility with pre- carious bone health, this can have major additive adverse effects on bone accrual and maintenance. Therefore, if depot progestogen is to be considered, it must be given with additional oestrogen. In turn, this probably should be via a transdermal patch, particularly where anticonvulsant use is concomitant and where oral oestrogen can be difficult to manage.

The implantable low-dose progestogen rod should not be used in a girl with a disability. For those who have major problems of understanding, insertion requires significant amounts of local anaesthetic $(5 \mathrm{ml})$ and is both painful and anxiety-provoking. For those who largely have an intellectual problem, such as autism, the patient is likely to pick at the palpable rod to try and remove it. In addition, the progestogen rod only causes total amenorrhoea for $10 \%$ of recipients. Another $10 \%$ have polymenorrhoea incurring need for removal and around $80 \%$ continue to have light periods.

The progestogen-bearing IUD has been very successful in adolescent girls with a disability [38]. Its advantages include 5 years of amenorrhoea and simultaneous contraception. It requires a brief general anesthetic for insertion for this type of adolescent, with a prerequisite for adult uterine dimensions. However it is rarely extruded. Usefully, this possibility can be observed in a patient who uses nappies (diapers).

Hysterectomy is rarely considered and only as a last resort if all other therapies have failed. Controversial ethical issues surround its use. In many countries a family law court decision would need to be made before it were permissible [39].

\section{Late Puberty}

Late puberty commonly occurs in those children with major disability who are orally fed, with low fat stores and inadequate nutrition.

Multiple pituitary hormone deficiencies or at least gonadotrophin deficiency should be considered if midline structural abnormalities co-exist.

Parents may perceive late puberty to be advantageous in the hope that the child may remain 'child-like' for longer and to provide less challenge for the family [40]. Disadvantages of this course of action clearly outweigh any perceived advantage, with late bone accrual giving prolonged high fracture risk in small, gracile bones. Late brain maturity and cooperation can also have an added adverse effect which is often not recognized by families.

Encouragement of spontaneous pubertal entry and progress within the normal age range expected for peers is desirable, for similar reasons. The majority of parents 
of children with major disabilities have fears that are not substantiated by events.

\section{Induction of Puberty}

For a child with extreme pubertal delay, induction of puberty may be beneficial. Where available, transdermal oestrogen for girls or testosterone for boys can be used, with slowly increasing doses to mimic normal puberty. For a girl, later addition of progestogen for 14 days every third calendar month to reduce frequency of endometrial shedding may be helpful. Generally, after puberty is complete, the adolescent will be able to sustain ongoing sex hormone production independently [41].

In view of the complex issues surrounding pubertal management in a child with disability, we undertook a study to identify an optimal method for addressing parental and carer education. Despite a well-publicized campaign through a large number of schools for children with a disability, attendance at meetings was low, suggesting that there may be domestic difficulties as a barrier to attendance. It is possible that there is less concern by parents than we had considered but our experience of families with a child with this type of problem suggests that knowledge is minimal and information is both helpful and required [42].

We further explored the issue, undertaking an education survey for medical staff including general paediatri- cians, general practitioners and paediatric endocrinologists as to their prescribing preferences and competence in dealing with management of menstrual disorders in adolescents with disability. There was a significant discrepancy in our findings between stated order of importance of choices for management and treatments prescribed. A low confidence level with high referral rates was prevalent amongst general and community paediatricians, indicating that further provision of education on this topic is needed [43].

\section{Conclusions}

The consequences of disability are life-long and have a global impact on the affected child and the family. Endocrine problems cover many areas of health management for these children. Assessment and management of puberty and bone health have particular impact on lifetime health and well-being. The paediatrician must be aware of a range of management options to improve outcomes.

\section{Disclosure Statement}

The author has no conflicts of interest to disclose.

\section{References}

1 Disease incidence, prevalence and disability'. Global Burden of Disease. World Health Organization. 2004. http://www.who.int/healthinfo/global_burden_disease/GBD_report_2004.

2 Zacharin M: Disorders of ovarian function in childhood and adolescence: evolving needs of the growing child. An endocrine perspective. BJOG 2010;117:156-162.

-3 Siddiqi SU, Van Dyke DC, Donohoue P, McBrien DM: Premature sexual development in individuals with neurodevelopmental disabilities. Dev Med Child Neurol 1999;41:392-395.

-4 Davies JH, Evans BA, Gregory JW: Bone mass acquisition in healthy children. Arch Dis Child 2005;90:373-378.

5 Seeman E: Pathogenesis of bone fragility in women and men. Lancet 2002;359:18411850.

6 Zacharin M: Assessing the skeleton in children and adolescents with disabilities: avoiding pitfalls, maximizing outcomes. A guide for the general paediatrician. J Paediatr Child Health 2009;45:326-331.
Houlihan CM, Stevenson RD: Bone density in cerebral palsy. Phys Med Rehabil Clin N Am 2009;20:493-508.

8 Modlesky CM, Subramanian P, Miller F: Underdeveloped trabecular bone microarchitecture is detected in children with cerebral palsy using high-resolution magnetic resonance imaging. Osteoporos Int 2008;19:169-176.

-9 Zacharin M: Current advances in bone health of disabled children. Curr Opin Paediatr 2004;16:545-551.

10 Kilpinen-Loisa P, Paasio T, Soiva M, Ritanen UM, Lautala P, Palmu P, Pihko H, Mäkitie O: Low bone mass in patients with motor disability: prevalence and risk factors in 59 Finnish children. Dev Med Child Neurol 2010;52: 276-282.

11 Hamed SA: Influences of bone and mineral metabolism in epilepsy. Expert Opin Drug Saf 2011;10:265-280.

12 Murray Houlihan C, Stevenson R: Bone density in cerebral palsy. Phys Med Rehabil Clin N Am 2009;20:493-508.
13 Coppola G, Fortunato D, Mainolfi C, Porcaro F, Roccaro D, Signoriello G, Operto FF, Verrotti A: Bone mineral density in a population of children and adolescents with cerebral palsy and mental retardation with or without epilepsy. Epilepsia 2012;53:2172-2177.

14 Henderson RC, Gilbert SR, Clement ME, Abbas A, Worley G, Stevenson RD: Altered skeletal maturation in moderate to severe cerebral palsy. Dev Med Child Neurol 2005;47:229236.

15 Stevenson RD, Conaway M, Barrington JW, Cuthill SL, Worley G, Henderson RC: Fracture rate in children with cerebral palsy. Pediatr Rehabil 2006;9:396-403.

-16 Verrotti A, Coppola G, Parisi P, Mohn A, Chiarelli $\mathrm{F}$ : Bone and calcium metabolism and antiepileptic drugs. Clin Neurol Neurosurg 2010;112:1-10.

$\checkmark 17$ Harcke HT, Taylor A, Bachrach S, Miller F, Henderson RC: Lateral femoral scan: an alternative method for assessing bone mineral density in children with cerebral palsy. Pediatr Radiol 1998;28:241-246.
Endocrine Problems in Children and Adolescents Who Have Disabilities 
18 Popp AW, Windolf M, Senn C, Tami A, Richards RG, Brianza S, Schiuma D: Prediction of bone strength at the distal tibia by HR-pQCT and DXA. Bone 2012;50:296-300.

19 Fricke O, Schoenau E: 'The Functional Muscle Bone Unit:' probing the relevance of mechanical signals for bone development in children and adolescents. Growth Horm IGF Res 2007;17:1-9.

20 Ward K, Alsop C, Caulton J, Rubin C, Adams J, Mughal Z: Low magnitude mechanical loading is osteogenic in children with disabling conditions. J Bone Miner Res 2004;19: 360-369.

-21 Gilsanz V, Wren TA, Sanchez M, Dorey F, Judex S, Rubin C: Low-level, high-frequency mechanical signals enhance musculoskeletal development of young women with low BMD. J Bone Miner Res 2006;21:1464-1474.

22 Caulton JM, Ward KA, Alsop CW, Dunn G, Adams JE, Mughal MZ: A randomised controlled trial of standing programme on bone mineral density in non-ambulant children with cerebral palsy. Arch Dis Child 2004;89: 131-135.

23 Stark C, Nikopoulou-Smyrni P, Stabrey A, Semler O, Schoenau E: Effect of a new physiotherapy concept on bone mineral density, muscle force and gross motor function in children with bilateral cerebral palsy. J Musculoskelet Neuronal Interact 2010;10:151158.

24 Henderson RC, Lark RK, Gurka MJ, Worley G, Fung EB, Conaway M, Stallings VA, Stevenson RD: Bone density and metabolism in children and adolescents with moderate to severe cerebral palsy. Pediatrics 2002;110:e5.

25 Henderson RC: Bone density and other possible predictors of fracture risk in children and adolescents with spastic quadriplegia. Dev Med Child Neurol 1997;39:224-227.
26 Binkley T, Johnson J, Vogel L, Kecskemethy $\mathrm{H}$, Henderson R, Specker B: Bone measurements by peripheral quantitative computed tomography (pQCT) in children with cerebral palsy. J Pediatr 2005; 147:791-796.

27 Zacharin M, Savasi I, Grover S: The impact of menstruation in adolescents with disabilities related to cerebral palsy. Arch Dis Child 2010; 95:526-530.

28 Hamilton A, Marshal MP, Sucato GS, Murray PJ: Rett syndrome and menstruation. J Pediatr Adolesc Gynecol 2012;25:122-126.

29 Hamilton A, Marshal MP, Murray PJ: Autism spectrum disorders and menstruation. J Adolesc Health 2011;49:443-445.

30 Burke LM, Kalpakjian CZ, Smith YR, Quint $\mathrm{EH}$ : Gynecologic issues of adolescents with Down syndrome, autism, and cerebral palsy. J Pediatr Adolesc Gynecol 2010;23:11-15.

31 Zacharin MR: Puberty, contraception, and hormonal management for young people with disabilities. Clin Pediatr (Phila) 2009;48: 149-155.

32 Grover SR: Gynaecological issues in adolescents with disability. J Paediatr Child Health 2011;47:610-613.

33 Quint EH: Menstrual issues in adolescents with physical and developmental disabilities. Ann NY Acad Sci 2008;1135:230-236.

34 Van Schrojenstein Lantman-de Valk HM, Rook F, Maaskant MA: The use of contraception by women with intellectual disabilities. J Intellect Disabil Res 2011;55:434-440.

- 35 Grover SR: Menstrual and contraceptive management in women with an intellectual disability. Med J Aust 2002;176:108-110.
6 Beksinska ME, Kleinschmidt I, Smit JA, et al: Bone mineral density in a cohort of adolescents during use of norethisterone enanthate, depot-medroxyprogesterone acetate or combined oral contraceptives and after discontinuation of norethisterone enanthate. Contraception 2009;79:345-349.

37 Arvio M, Kilpinen-Loisa P, Tiitinen A, Huovinen K, Mäkitie O: Bone mineral density and sex hormone status in intellectually disabled women on progestin-induced amenorrhea. Acta Obstet Gynecol Scand 2009;88: 428-433.

38 Pillai M, O’Brien K, Hill E: The levonorgestrel intrauterine system (Mirena) for the treatment of menstrual problems in adolescents with medical disorders or physical or learning disabilities. BJOG 2010;117:216-221.

39 Isaacs D, Tobin B, Hamblin J, Slaytor E, Donaghue KC, Munns C, Kilham HA: Managing ethically questionable parental requests: growth suppression and manipulation of puberty. J Paediatr Child Health 2011;47: 581-584.

40 Ashley's blog: http://ashleytreatment.spaces. live.com/blog.

41 Gold MA, Duffy K: Extended cycling or continuous use of hormonal contraceptives for female adolescents. Curr Opin Obstet Gynecol 2009;21:407-411.

42 Neylon O, Grover S, Zacharin M: Carers' concerns regarding menstruation in girls with an intellectual disability. ESPE Annual Meeting, Leipzig 2012. Horm Res Paediatr 2013, submitted.

43 Neylon O, Grover S, Zacharin M: Exploring clinician confidence in the management of adolescent menstrual and reproductive health problems. ESPE Annual Meeting, Leipzig 2012. Horm Res Paediatr 2013, submitted. 